\title{
Full Dynamic Range Energy Calibration of CALET onboard the International Space Station
}

\author{
Ryohei Miyata* \\ Graduate School of Advanced Science and Engineering, Waseda University \\ E-mail: miya6236eruri.waseda.jp
}

\section{Yoichi Asaoka}

Research Institute for Science and Engineering, Waseda University

E-mail: yoichi.asaoka@aoni.waseda.jp

\author{
Shoji Torii \\ School of Advanced Science and Engineering, Waseda University and Research Institute for \\ Science and Engineering, Waseda University \\ E-mail: torii.shoji@waseda.jp
}

\section{for the CALET collaboration}

In August 2015, the CALET (CALorimetric Electron Telescope) instrument, designed for long exposure observations of high energy cosmic rays, docked with the ISS (International Space Station) and shortly thereafter began to collect data. CALET will measure the cosmic ray electron spectrum over the energy range of $1 \mathrm{GeV}$ to $20 \mathrm{TeV}$ with a very deep calorimeter with both total absorption and imaging (TASC and IMC) units. Each TASC readout channel must be carefully calibrated over the extremely wide dynamic range of CALET that spans six orders of magnitude to obtain a degree of precision necessary to achieve high energy resolution. The entire dynamic range is covered by four different gain ranges, based on two photon detectors - an avalanche photodiode (APD) and a regular photodiode (PD) - in conjunction with a shaper amplifier with two gain ranges. The energy calibration process consists of three steps. First step is determination of conversion factor between ADC units and the energy deposit. By this way, the conversion factor of APD high gain is determined. Next step is linearity measurements over each gain range. UV pulse laser calibrations were performed on ground to confirm this linearity. Third step is correlation measurements between adjacent gain ranges. After confirming the input-output linearity of each readout channel of TASC, using the conversion factor of APD high and correlations, the conversion factor of all readout channels can be determined. Finally, using the estimated calibration errors and measured detector responses, such as the pedestal noise, the errors in the energy deposit sum were calculated for simulated electron events from $1 \mathrm{GeV}$ to $20 \mathrm{TeV}$. As a result, $2 \%$ precision level energy calibration was achieved over the entire dynamic range above $10 \mathrm{GeV}$.

35th International Cosmic Ray Conference - ICRC2017

10-20 July, 2017

Bexco, Busan, Korea

${ }^{*}$ Speaker. 


\section{Introduction}

The CALET (CALorimetric Electron Telescope) [1] was docked to Exposed Facility of the Japanese Experiment Module (JEM-EF) on the International Space Station (ISS) in August 2015 and has been collecting data [2] since October 2015. It has been designed for long duration observations of high energy cosmic rays onboard the ISS. The instrument consists of two layers of segmented plastic scintillators for the cosmic-ray charge identification (CHD), a 3 radiation length thick tungsten-scintillating-fiber imaging calorimeter (IMC), and a 27 radiation length thick leadtungstate calorimeter (TASC). The primary purpose of CALET is to discover nearby cosmic-ray sources and to search for dark matter with precision measurements of electron and gamma ray spectra over a wide energy range. The calorimeter provides efficient absorption of the electron shower energy in the TeV energy range and is able to identify electrons in the presence of a very high proton flux, with a rejection factor of $10^{5}$ based on the difference in shower development. This instrument will therefore be used to acquire the cosmic ray electron spectrum over the energy range of $1 \mathrm{GeV}$ to $20 \mathrm{TeV}$ with exceptional energy resolution, especially above $100 \mathrm{GeV}$, where the resolution is better than $2 \%$. Since each channel of the TASC has a dynamic range of six orders of magnitude, CALET is capable of determining the energy of primary particles from $1 \mathrm{GeV}$ to $1 \mathrm{PeV}$. This enables the instrument to measure proton and nuclei spectra as well as electron and gamma ray spectra over this extremely wide energy range. The present paper details the calibration method in full dynamic range to accomplish the high precision observation [3].

\section{Energy Measurement and Calibration Method}

\subsection{Energy calibration in full dynamic range}

The TASC has an overall depth of $27 X_{0}$ and consists of 12 detection layers in an alternating orthogonal arrangement, each comprised of 16 lead tungstate crystal $\left(\mathrm{PbWO}_{4}\right.$ or PWO) logs with dimensions of $2.0 \times 1.9 \times 32.6 \mathrm{~cm}^{3}$. As a result of this design, the TASC is able to image the development of the shower in three dimensions. With the exception of the first layer which uses PMTs, a photodiode (PD) in conjunction with an avalanche photodiode (APD) reads the photons generated by each PWO log. The entire dynamic range is covered by four different gain ranges, based on two photon detectors, an APD and a PD, in conjunction with a shaping amplifier with lower and higher gains. Employing dual shaping amplifiers with two different gains for each APD and PD increases the dynamical range to $10^{6}$. Similarily, dual shaping amplifiers with two gains are also used with the PMTs, which increases the dynamical range to $10^{4}$. Careful calibration of each TASC readout channel is required to achieve a sufficient accuracy that matches the intrinsic energy resolution over the wide dynamic range of six orders of magnitude.

Figure 1 explains the energy calibration processes. At first, calibration with minimum ionizing particles (MIPs) is adopted to get a conversion factor from ADC value to the absolute energy deposit by non-interacting particles, protons or helium, by using the high gain APD range. Then the conversion factor between ADC value by APD with high gain and the energy is determined. Next, the linearity in each gain range is examined. Prior to launch, the linearity was confirmed by onground calibration using a UV pulse laser, during which the APD and PD outputs were determined as a function of the laser energy. The third step is measurement of the correlation between adjacent 

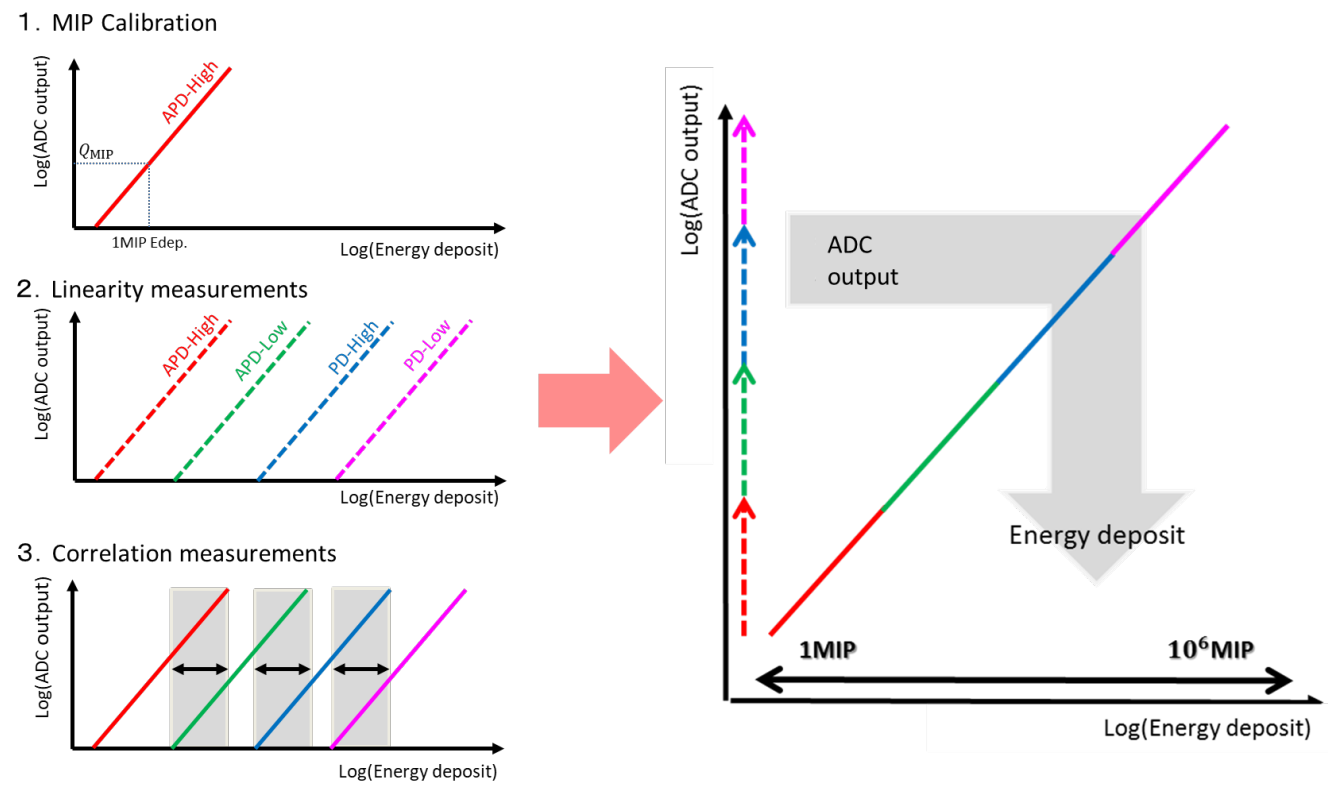

Figure 1: The conceptual diagram of the energy calibration processes.

gain ranges. Taking advantage of the nearly one order of magnitude overlap between the adjacent gain ranges, it was possible to measure identical energy deposits at two gain ranges. As the linearity of each gain range had already been confirmed, the gain over the entire dynamic range could be determined based on the ADC-to-energy conversion factor, using the acquired gain ratios between adjacent gain ranges. Details of the MIP calibration of CALET is given in Ref. [5]. In this paper, the following steps of the calibration are described.

\subsection{Linearity Measurements over the Entire Dynamic Range}

It is necessary to determine the input-output relationship over each gain range with groundbased measurements prior to launch Therefore, the relative gain change between the four ranges is monitored, and is corrected using gain ratio measurements, as explained in the following section. UV pulse laser calibrations were performed on ground for linearity confirmation. While scanning the pulse laser intensity through six orders of magnitude, detailed measurements were made of the four APD/PD output responses from each of the 176 PWO logs.

Figure 2 presents a schematic diagram of the UV pulse laser test of each PWO. The PWO was irradiated from one end, and the emitted photons were detected with the APD/PD at the opposite end. By combining four readouts, the full dynamic range of six orders of magnitude is covered while maintaining a nearly one order of magnitude overlap between adjacent gain ranges. It should be noted that there is crosstalk from the APD to the PD due to stray capacitance between these two devices. When a charge sensitive amplifier (CSA) of the APD is saturated, the feedback from the CSA becomes insufficient and the potential at the APD-CSA input has a non-zero value. Although the crosstalk amounts to only $\sim 0.1 \%$ of the charge ratio, it can become significant due to the APDPD gain/area ratio of 1000 to 1 (APDs have a 20 times larger area and a 50 times higher gain). 


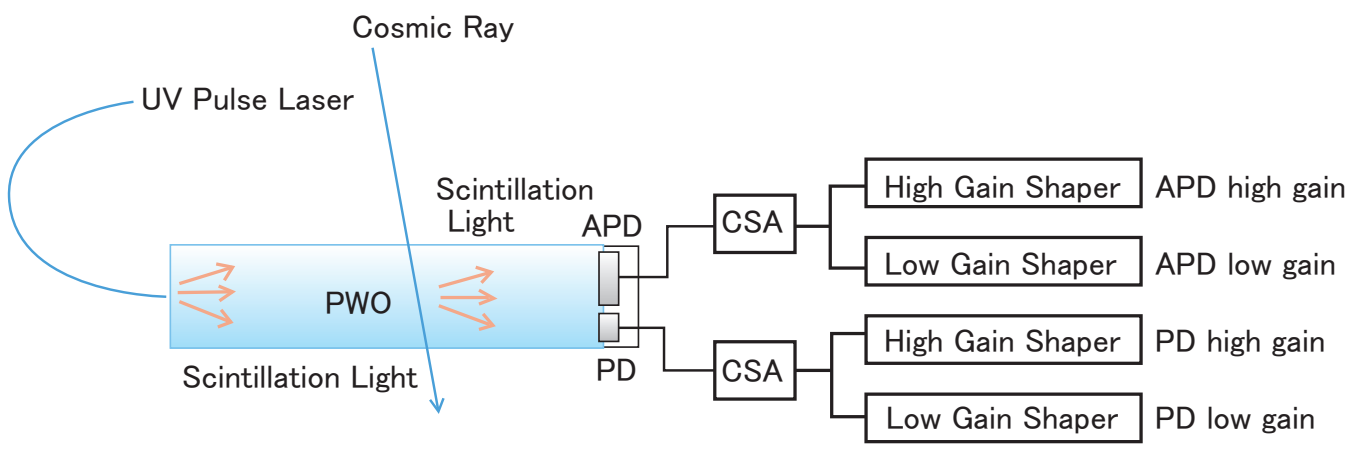

Figure 2: Schematic view of UV pulse laser light injection into the PWO, together with TASC APD/PD readouts.
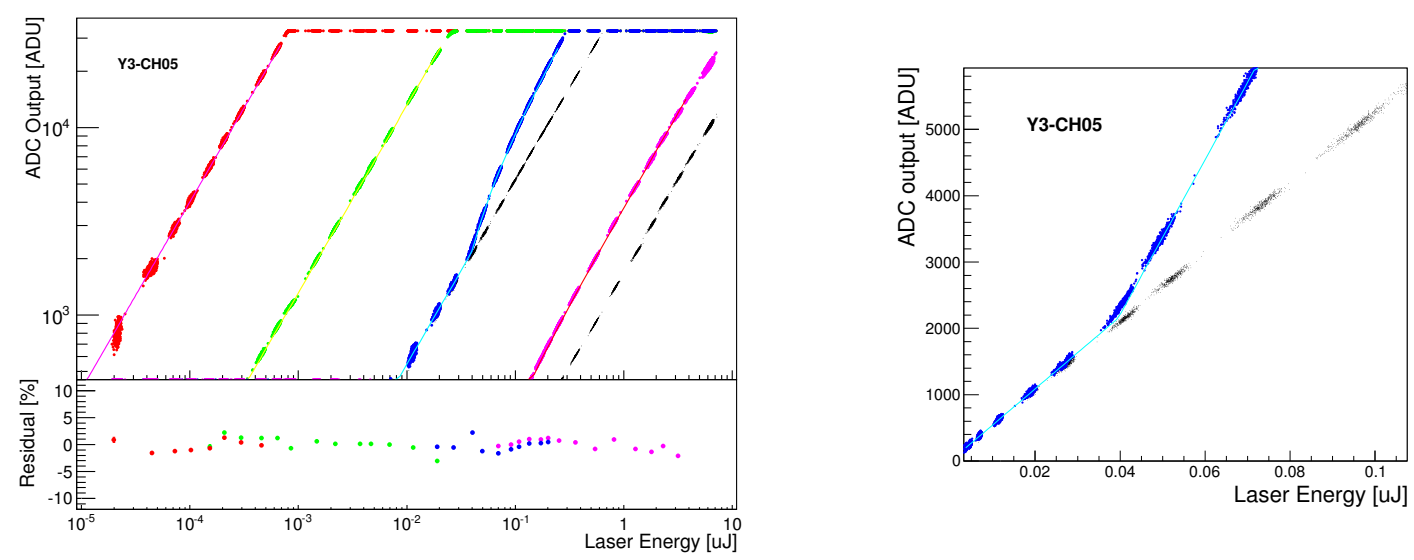

Figure 3: Typical data acquired from UV pulse laser calibration. (Left) From left to right, the responses of the APD high gain (red data points), APD low gain (green), PD high gain (blue) and PD low gain (magenta) are plotted as a function of laser energy with the residuals from fitted functions shown in the bottom panel. Black points in the PD range represent data acquired without an APD bias. (Right) Close up view of the PD high gain response, applying a linear scale to better demonstrate the simple broken linear relationship.

Since the crosstalk signal is proportional to the input charge and is stable, it is possible to calibrate the input-output relationship using UV pulse laser data.

Figure 3 shows an example of the data obtained from UV pulse laser measurements. Here, the horizontal and vertical axis represent the laser energy and ADC values, respectively. Since the laser energy is monitored on a pulse-by-pulse basis, the linearity over the entire dynamic range was confirmed using 17,000 points of laser pulse data for each channel. As a result of the APD/PD crosstalk, the PD response exhibits a slope break corresponding to the APD-CSA saturation point, as shown in the right panel of Fig. 3. The responses of all APD/PD channels were measured and the data confirmed the linear and broken linear relationships of the APD and PD response functions, respectively, as a result of fitting of the data points with appropriate functions for each range. To estimate the errors resulting from fitting the linear and broken linear functions, the distributions of residuals from the fitting functions were assessed for each gain range. The errors were estimated to 
be $1.4 \%, 1.5 \%, 2.5 \%$ and $2.2 \%$ for the APD high, APD low, PD high and PD low gain, respectively from the RMS of the distributions. Although these errors include both possible nonlinearities and expected statistical variations in measurements, in addition to UV laser system calibration errors, we adopted these values as the actual errors due to possible non-linear effects.

It is expected that both the APD gain and the PWO light yield will vary between conditions on-ground and those onboard the ISS, as well as with time during on-orbit observations. This corresponds to a change in the amount of crosstalk charge per unit energy deposit and thus results in a slope change in the APD/PD crosstalk region. We confirmed this effect using UV laser data acquired at a higher APD bias ( $\sim \times 2$ gain). During this laser calibration process, three data sets with different APD gains (nominal gain, $\sim \times 2$ gain and small gain without APD bias) were obtained to validate our simple model for correcting APD/PD crosstalk and to estimate the correction errors, as well as to calibrate all the gain ranges. This effect is revisited in the next section. in relation to gain correlation measurements.

\subsection{Cross Calibration of Adjacent Gain Ranges}

The gain correlations between adjacent gain ranges were used to correct for possible gain changes between the UV laser calibrations performed on the ground and observations onboard the ISS. Figure 4 presents examples of gain ratio measurements in the APD high gain to APD low gain
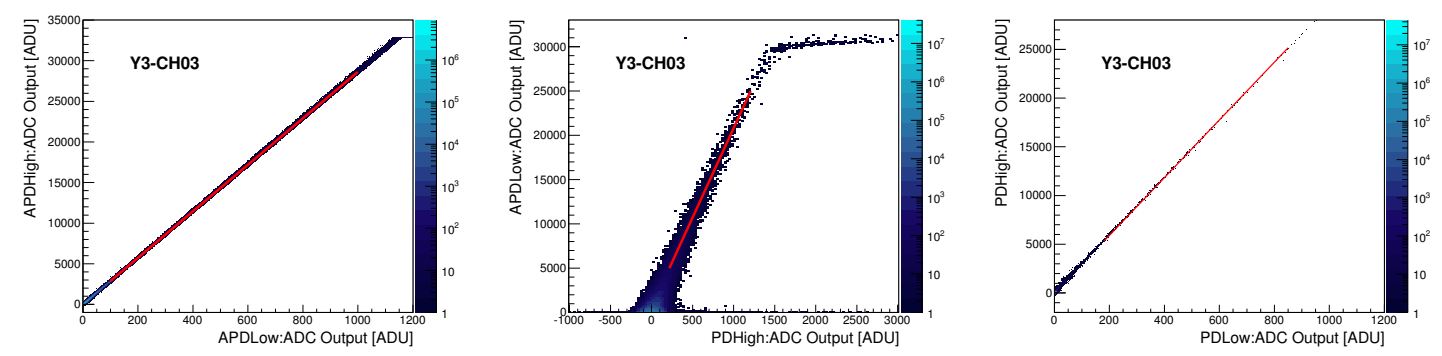

Figure 4: Typical gain correlation measurements between adjacent gain ranges. (Left) APD high gain to APD low gain, (Middle) APD low gain to PD high gain, and (Right) PD high gain to PD low gain regions.

(Left), APD low gain to PD high gain (Middle), and PD high gain to PD low gain (Right) regions. In the fitting of each channel, proper selection of the fitting range was vital to avoid saturation effects in the higher gain range and the lower signal-to-noise region due to pedestal noise in the lower gain range. While the offset was set to zero in most cases, non-zero offsets during linear fitting were allowed in some cases involving PD-high to APD-low gain ratio fitting due to APD-to-PD crosstalk prior to APD-CSA saturation. In such cases, correct treatment was ensured by using the same offset during linear fitting of the UV pulse laser data. The errors on the gain ratios were determined from the parameter errors in the linear fittings since the reduced chi-squared distributions were found to be reasonable, having average values of approximately 1 . The errors on the ratios were found to be $0.1 \%, 0.7 \%$ and $0.1 \%$ for the APD high gain to APD low gain, PD high gain to APD low gain and PD high gain to PD low gain regions, respectively. Since the linearity of each gain range is confirmed by UV laser tests, calibration over the whole dynamic range is now possible by applying the conversion factor to the subsequent gain range using the gain ratios. 
As explained in the previous section, slope changes in the on-orbit calibration of the APD range with respect to the ground data were foreseen due to the different environment experienced in orbit. This also affects the APD-to-PD crosstalk region, which was corrected based on the assumption that the slope change in the PD range after APD-CSA saturation is proportional to the slope change in the APD range between ground and orbit. When applying such corrections, it is important to identify the crosstalk component because the slope associated with the PD gain is not affected by the APD gain change. UV laser data acquired with a $\sim \times 2$ gain were used to validate the correction method and to estimate the associated errors. By applying the same procedure to $\sim \times 2$ gain data and comparing the predicted slope with the measured slope in the PD high gain range above APD-CSA saturation point, we were able to estimate the errors associated with our simple model for the correction of the APD-PD crosstalk effect. When applying this method to on-orbit data, the error was scaled to the actual gain difference, and the resultant error on the gain was estimated to be $1.1 \%$. Since it is not possible to determine this error from the on-orbit data, we consider this error to represent a systematic uncertainty on the energy scale as well as an estimation of the calibration error that affects the energy resolution.

As a result, we have obtained an energy deposit distribution which is extended to about $1 \mathrm{PeV}$ in six orders of magnitude of energy as shown in left panel of Figure 5.
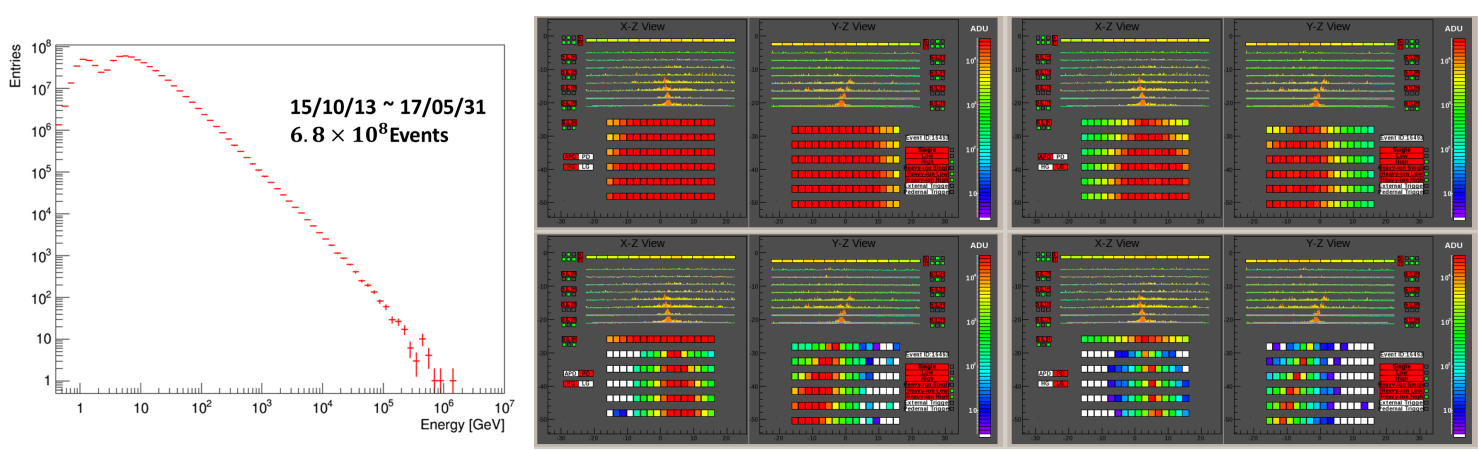

Figure 5: Left panel shows TASC energy deposit sum spectrum during October 13, 2015 to May 31, 2017. Right panel represents the highest energy event penetrating whole detectors (about $1 \mathrm{PeV}$ ). In this panel, top left shows APD high gain view, top right APD low gain, bottom left PD high gain and bottom right PD low gain respectively. It is clearly seen that the four gains cover a whole range of the energy measurements.

\section{Energy Measurement: Error and Resolution}

Using the estimated calibration errors and measured detector responses, such as the pedestal noise on a channel-by-channel basis, the errors in the energy deposit sum were calculated for simulated electron events from $1 \mathrm{GeV}$ to $20 \mathrm{TeV}$ (see Ref. [3] for details). The left panel of Fig. 6 presents the energy dependence of the relative error in the energy deposit sum measurements. As clearly shown by this figure, a $2 \%$ precision level energy calibration was achieved over the entire dynamic range above $10 \mathrm{GeV}$. The reduced accuracy with which the energy deposit can be determined below $10 \mathrm{GeV}$ is due to pedestal noise. As reported in detail in Ref. [4], the requirements for the calibration error of each TASC $\log$ can be relaxed by a factor of $\sim 3$ compared to that for 

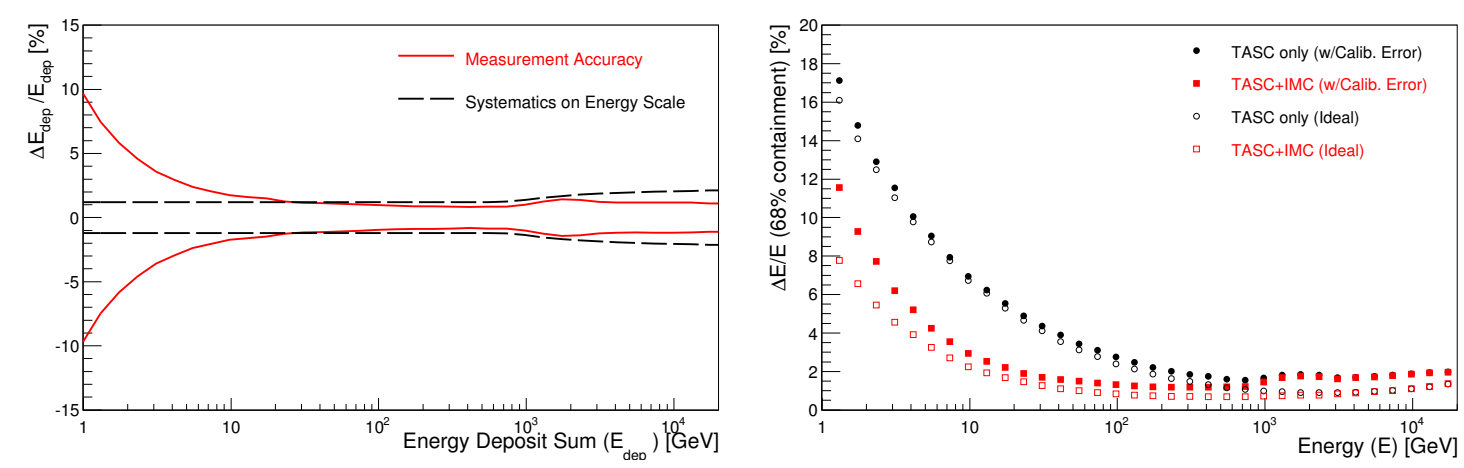

Figure 6: (Left) Energy dependence of the relative error in the energy deposit sum measurements for electrons, considering all the energy calibration errors and detector responses (solid red lines). The systematic uncertainty on an absolute scale is also shown by black dashed lines. (Right) Estimated energy resolution for electrons as a function of energy. Open red squares denote intrinsic resolution and closed red squares denote actual resolution including all the detector responses and calibration errors in the case of energy determination using the TASC+IMC. Circular symbols indicate energy determination using the TASC only.

the energy resolution, as long as these individual errors of in total $\sim 6 \%$ are randomly distributed. This is due to the fact that, on average, $\sim 10$ TASC logs contribute significantly to an event's energy measurement. The results obtained here are therefore perfectly consistent with the expected values. The estimated systematic uncertainty is also plotted on an absolute scale in Fig. 6. The systematic uncertainty in the energy scale was estimated to be less than $\sim 2 \%$. Since the calibration error is a fixed value for each channel, there could be systematic bias on the energy measurements. To account for this effect, several sets of simulation data were generated and evaluated for such a systematic bias. It should be noted that the PD range becomes important, i.e., accounts for more than $20 \%$ of an energy measurement, at an energy deposit sum of $1 \mathrm{TeV}$, resulting in slightly larger systematic uncertainties in this range, although the calibration accuracy is still satisfactory. Furthermore, improvement in our knowledge of the systematic uncertainty on the energy scale is expected as long as the collected data statistics grow, which will allow us to understand the detector better.

\section{Conclusion}

Energy calibration of the CALET, launched to the ISS in August 2015 and accumulating scientific data since October 2015, was performed using both flight data and calibration data acquired on the ground before launch. By taking advantage of the fully-active total absorption calorimeter, absolute calibration between ADC units and energy was possible with an accuracy of a few percent, using penetrating particles. Successful calibration was achieved over the complete dynamic range of six orders of magnitude for each TASC channel with sufficient accuracy to maintain a fine resolution of $2 \%$ above $100 \mathrm{GeV}$ by combining two calibration processes: linearity measurements and determination of the correlation between adjacent gain ranges. The systematic error in the energy scale was also estimated based on the calibration results and was found to be $\leq 2 \%$. Additionally, since the long term stability of the conversion factor of APD high gain has been confirmed by MIP calibration, stable measurements over the full dynamic range can be accomplished. 


\section{Acknowledgements}

We gratefully acknowledge JAXA's contributions to the development of CALET and to operations on board the ISS. We also wish to express our sincere gratitude to ASI and NASA for their support of the CALET project. Finally, this work was partially supported by a JSPS Grant-in-Aid for Scientific Research (S) (no. 26220708) and by the MEXT-Supported Program for the Strategic Research Foundation at Private Universities (2011-2015) (no. S1101021) at Waseda University.

\section{References}

[1] S. Torii for the CALET Collaboration, "The CALorimetric Electron Telescope (CALET): High Energy Astroparticle Physics Observatory on the International Space Station”, Proceedings of Science, Proc. of the 34th ICRC (The Hague, Netherlands), 581

[2] Y. Asaoka for the CALET Collaboration, "Development of the Waseda CALET Operations Center (WCOC) for Scientific Operations of CALET", Proceedings of Science, Proc. of the 34th ICRC (The Hague, Netherlands), 603.

[3] Y. Asaoka for the CALET Collaboration, "Energy Calibration of CALET Onboard the International Space Station “, Astroparticle Physics. 91 (2017) 1-10.

[4] T. Niita et al., "Energy Calibration of Calorimetric Electron Telescope (CALET) in Space", Adv. Space Res. 55 (2015) 2500.

[5] Y. Komiya for the CALET Collaboration, "MIP Calibration and the Long-term Stability of CALET Onboard the International Space Station“, Proceedings of the 35th ICRC (Busan, Korea), PoS (ICRC2017) 206(2017). 